Original Paper

\title{
Exercise Training Attenuates Proinflammatory Cytokines, Oxidative Stress and Modulates Neurotransmitters in the Rostral Ventrolateral Medulla of Salt- Induced Hypertensive Rats
}

\author{
Hong-Bao Lia Chan-Juan Huo ${ }^{a}$ Qing Su ${ }^{a}$ Xiang Lia Juan Baia Guo-Qing Zhub \\ Yu-Ming Kang ${ }^{a}$ \\ aDepartment of Physiology and Pathophysiology, Key Laboratory of Environment and Genes Related \\ to Diseases, Xi'an Jiaotong University School of Basic Medical Sciences, Xi'an Jiaotong University \\ Health Science Center, Xi'an Jiaotong University Cardiovascular Research Center, Xi'an, ${ }^{\circ} K e y$ Laboratory \\ of Cardiovascular Disease and Molecular Intervention, Department of Physiology, Nanjing Medical \\ University, Nanjing, China
}

\section{Key Words}

Exercise training $\cdot$ Rostral ventrolateral medulla $\cdot$ Neurotransmitters $\cdot$ Nuclear factor-kappa B - Oxidative stress $\cdot$ Hypertension

\begin{abstract}
Background/Aims: Exercise training (ExT) was associated with cardiovascular diseases including hypertension. The rostral ventrolateral medulla (RVLM) is a key region for central control of blood pressure and sympathetic nerve activity. Therefore, this study aimed to investigate the mechanisms within RVLM that can influence exercise training induced effects in salt-induced hypertension. Methods: Male Wistar rats were fed with a normal salt (0.3\%) (NS) or a high salt (8\%) (HS) diet for 12 weeks to induce hypertension. Then these rats were given moderate-intensity ExT for a period of 12 weeks. RVLM was used to determine glutamate and gamma-aminobutyric acid (HPLC), phosphorylated IKK $\beta$, Fra-LI, 67-kDa isoform of glutamate decarboxylase (GAD67), proinflammatory cytokines (PIC) and NADPH-oxidase (NOX) subunits expression (Immunohistochemistry and Immunofluorescence, Western blotting). PIC and NF-KB p65 activity in the plasma were evaluated by ELISA studies. Renal sympathetic nerve activity (RSNA) was recorded and analyzed using the PowerLab system. Results: High salt diet resulted in increased mean arterial pressure and cardiac hypertrophy. These high salt diet rats had higher RVLM levels of glutamate, PIC, phosphorylated IKK $\beta$, NF-KB p65 activity, Fra-LI, superoxide, NOX- 2 (gp91 ${ }^{\text {phox }}$ ) and 4, and lower RVLM levels of gamma-aminobutyric acid and GAD67, and higher plasma levels of PIC, norepinephrine, and higher RSNA. ExT attenuated

\begin{tabular}{ll}
\hline Yu-Ming Kang, M.D., Ph.D. & Department of Physiology \& Pathophysiology, Xi'an Jiaotong University School of Medicine \\
& Xi'an 710061, (China) \\
& Tel. +86 2982657677, Fax +86 2982657677, E-Mail ykang@mail.xjtu.edu.cn
\end{tabular}
\end{abstract}


Li et al.: Exercise Training in Salt-Induced Hypertension

these changes in salt-induced hypertensive rats. Conclusions: These findings suggest that high salt diet increases the activity of NF-KB and the levels of PIC and oxidative stress, and induces an imbalance between excitatory and inhibitory neurotransmitters in the RVLM. ExT attenuates hypertension and cardiac hypertrophy partially mediated by attenuating oxidative stress and modulating neurotransmitters in the RVLM.

\section{Introduction}

Hypertension is characterized by cardiac hypertrophy and dysfunction, and elevated levels of blood pressure (BP) and sympathetic tone [1]. It is well known that the rostral ventrolateral medulla (RVLM) is a main active region for central regulation of the cardiovascular function and plays a key role in maintaining resting BP and sympathetic tone $[2,3]$. A growing body of evidence indicates that abnormalities in structure and function of the RVLM contribute to the pathogenesis of hypertension $[3,4]$.

We and others have indicated that increased pro-inflammatory cytokines (PIC) such as tumour necrosis factor-alpha (TNF- $\alpha$ ), interleukin (IL)-1 $\beta$ and IL- 6 in the rostral ventrolateral medulla (RVLM) have a significant impact on sympathetic outflow, arterial pressure and cardiac remodeling in experimental models of hypertension [5]. High level of BP and sympathetic overactivity are also closely related to the enhancement of reactive oxygen species (ROS) in the RVLM under the hypertensive state [5, 6]. Over- production of ROS can activate several redox-sensitive signal transduction pathways such as nuclear factor-kappaB (NF- $\kappa \mathrm{B})$. Activation of NF- $\kappa \mathrm{B}$ induces transcription of PIC genes, leading to further increases in ROS production and fostering a cyclic positive feedback mechanism, thereby accelerating the progression of hypertension and its associated heart changes [7].

Exercise training (ExT) is an efficient strategy for the prevention and treatment of hypertension, and is acknowledged by all major institutes. It is reported that ExT effectively lowers BP, decreases cardiac output, and enhances the baroreflex sensitivity in patients [8] and animals $[6,9,10]$ with hypertension. However, the exact mechanisms of exerciseinduced effects in hypertension are still poorly understood. Previous studies often focused on the peripheral mechanisms which ExT may act on, such as reduced peripheral vascular resistance [11], reductions in oxidative stress [12] and predominance of endothelium relaxing over contractile factors [13].

Recent evidence suggested that neural plasticity in the central cardiovascular networks can be more important for the beneficial effects of ExT on cardiovascular dysfunctions [1417]. Growing evidence has been demonstrated that ExT significantly attenuates increases in BP and sympathetic activity induced by stimulation of the RVLM [18], suggesting the importance of the RVLM in mediating the effects of ExT on cardiovascular regulation. Therefore, this study was designed to investigate whether exercise training attenuates blood pressure, sympathetic nerve activity and cardiac hypertrophy by modulating proinflammatory cytokines, oxidative stress and neurotransmitters within RVLM in salt induced hypertension.

\section{Materials and Methods}

\section{Animals and Experimental Design}

Male Wistar rats were received at 6 weeks of age and were allowed to acclimate for 1 week with standard rat diet (Beijing Vital River Laboratory Animal Technology Co., Ltd, China) and tap water before beginning the experiment. Rats were housed at a constant room temperature, humidity and light cycle (12:12 h light-dark) with free access to tap water and fed with standard rat chow ad libitum. During the 24week experimental period, the NS group received a normal salt (0.3\%) diet (Beijing Vital River Laboratory Animal Technology Co., Ltd, China) and the HS group received a high salt (8\%) diet (Beijing Vital River Laboratory Animal Technology Co., Ltd, China). At the 12th week, these rats were randomly selected and 
received moderate-intensity exercise trained or sedentary for a period of 12 weeks. Therefore, 4 groups ( $\mathrm{n}=30 /$ group) were included in this study: (1) Wistar normal salt diet $(0.3 \% \mathrm{NaCl})+$ sedentary $(\mathrm{NS}+\mathrm{Sed})(2)$ Wistar normal salt $\operatorname{diet}(0.3 \% \mathrm{NaCl})+$ exercise trained (NS + ExT) (3) Wistar high salt diet $(8 \% \mathrm{NaCl})+$ sedentary $(\mathrm{HS}+\mathrm{Sed})(4)$ Wistar high salt diet $(8 \% \mathrm{NaCl})+$ exercise trained $(\mathrm{HS}+\mathrm{ExT})$ (Fig. 1). All animal and experimental procedures in this study were reviewed and approved by the Animal Care and Use Committees of Xi'an Jiaotong University and were conducted in accordance with the Guide for the Care and Use of Laboratory Animals (National Institutes of Health publication No. 85-23, revised 1996). At the end of the study, rats were euthanized; the blood, brains and heart tissues were collected, and immediately frozen on dry ice. Some rats ( $\mathrm{n}=21$ for each group) were used for hemodynamic and anatomical studies. The rostral ventrolateral medulla (RVLM) tissues were punched out from the brain for western blot analysis or for immunohistochemistry assay $(n=7$ for each group).

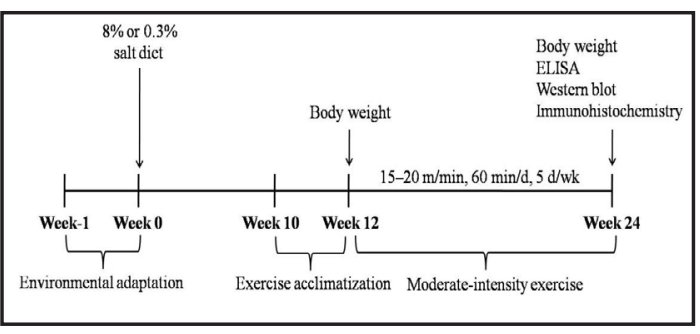

Fig. 1. Experimental Design. Six-week-old male Wistar rats were first acclimatized to the environment for 1 week before the start of the experiment. After 1 week of acclimation, the rats received a normal salt $(0.3 \%)$ diet or high salt $(8 \%)$ diet for 24 weeks. At the 10 th week, the rats were acclimatized to exercise trained for 2 week. At the 12th week, these rats were randomly selected and received moderate-intensity exercise trained or sedentary for a period of 12 weeks. At the 24th week, animals were weighed and the parameter was measured. The animals were then euthanized and the brains were collected for Immunohistochemistry and Western blot analysis.

\section{Exercise training protocol}

Exercise training was performed on a motor treadmill (FT-200, Taimen Co., Cheng du, China) for 12 weeks ( 5 days per week; 60 min per day, $0^{\circ}$ inclination), which includes an acclimation period of 2 weeks. After acclimation, training intensity was set at $55-65 \%$ of maximal aerobic velocity (MAV), which corresponds to moderate-intensity exercise $(15-20 \mathrm{~m} / \mathrm{min})$. This training intensity was maintained throughout the study period. To determine the MAV, rats were submitted to an incremental exercise test as reported previously $[15,19,20]$. The rats in sedentary groups (NS + Sed and HS + Sed) were placed on a nonmoving treadmill during the training sessions.

\section{Blood pressure measurements}

Blood pressure (BP) was measured in conscious animals by a tail-cuff occlusion method [21, 22]. BP was measured at baseline and then every 2 weeks until the end of the study period. Unanesthetized rats were warmed to an ambient temperature of $30^{\circ} \mathrm{C}$ by placing rats in a holding device mounted on a thermostatically controlled warming plate. Blood pressure values were averaged from at least seven consecutive cycles obtained from each rat.

\section{Hemodynamic analyses and Renal Sympathetic Nerve Recordings}

The preparation for measuring hemodynamic and renal sympathetic nerve activity (RSNA) parameters was performed as previously described $[23,24]$. Briefly, rats were anaesthetized with a ketamine (80 $\mathrm{mg} / \mathrm{kg}$ ) and xylazine $(10 \mathrm{mg} / \mathrm{kg}$ ) mixture (ip). The carotid artery and jugular vein were cannulated, and the arterial line was connected to a pressure transducer (Gould P23 1D) for data recording and analysis (PowerLab, AD Instruments, Castle Hill, Australia) of systolic blood pressure (SBP), diastolic blood pressure (DBP), and heart rate (HR). Next, a retroperitoneal incision was made and the left renal sympathetic nerve was isolated. The renal nerve was placed on a platinum electrode which is connected with the recording system and immersed in warm mineral oil. The electrical signal was amplified with high- and low-frequency cutoffs of 1, 000 and $100 \mathrm{~Hz}$, respectively (Grass amplifier). The rectified output from the amplifier (RC filtered, time constant: $0.5 \mathrm{~s}$ ) was displayed using the PowerLab system (8si, AD Instruments) to record and integrate the raw renal nerve discharge. Baseline recordings of RSNA, arterial blood pressure (ABR) and HR were taken for several minutes. Maximum RSNA was detected using an intravenous bolus administration 


\section{Cellular Physiology Cell Physiol Biochem 2018;48:1369-1381

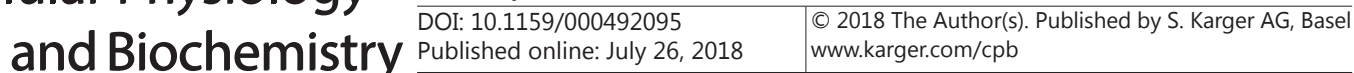

Li et al.: Exercise Training in Salt-Induced Hypertension

of sodium nitroprusside (SNP, $10 \mu \mathrm{g}$ ), which lowered ABP to between 45 and $50 \mathrm{mmHg}$. Arterial baroreflex control of RSNA was determined with the response of these parameters to an injection of SNP (10 $\mu \mathrm{g})$. The background noise defined as the signal-recorded post-mortem was subtracted from actual RSNA and expressed as percent of maximum (in response to SNP) at the end of the experiment. Because the maximum activity was similar with the SNP, we elected to use the response to SNP to determine maximum activity in this study.

Arterial baroreflex curves were constructed as we described previously [25]. In brief, several points for RSNA were taken during the fall in ABP after the administration of SNP. The logistic regression curve as described by Kent et al [26]. was fit to the data points with the following equation:

RSNA $=A_{1} /\left\{1+\exp \left[A_{2}\left(M A P-A_{3}\right)\right]\right\}+A_{4}$

Where $A_{1}$ is RSNA range, $A_{2}$ is the slope coefficient, $A_{3}$ is the pressure at the midpoint of the range, and $\mathrm{A}_{4}$ is minimum RSNA. The peak slope (or maximum gain) was determined by taking the first derivative of the baroreflex curve described by equation 1 . The first derivative is described by equation 2 .

Slope $=\left\{A_{1} \times A_{2} \times \exp \left[A_{2}\left(M A P-A_{3}\right)\right]\right\} /\left\{1+\exp \left[A_{2}\left(M A P-A_{3}\right)\right]\right\}^{2}$

The mean value of each curve parameter was used to derive a composite curve for each group of rats before and after each intervention.

\section{Collection of blood and tissue samples}

At the end of the study, rats were anesthetized with a ketamine $(80 \mathrm{mg} / \mathrm{kg})$ and xylazine $(10 \mathrm{mg} / \mathrm{kg})$ mixture (ip). Blood samples were collected from the abdominal aorta with heparin-moistened syringes in chilled EDTA tubes. The plasma samples were separated by centrifugation (3000x $g$ for $25 \min$ at $\left.4{ }^{\circ} \mathrm{C}\right)$ and stored at $-80^{\circ} \mathrm{C}$ until assayed for PIC and norepinephrine (NE) levels. The brain and heart were removed and harvested quickly. The heart weight/body weight (HW/BW) ratio was measured as previously described [21]. The RVLM tissue was punched from each brain according to the methods as previously described [4]. Briefly, rats were anesthetized with a ketamine $(80 \mathrm{mg} / \mathrm{kg})$ and xylazine $(10 \mathrm{mg} / \mathrm{kg})$ mixture (ip) and the brains were quickly removed, frozen, and stored at $-80{ }^{\circ} \mathrm{C}$ until use. Freshly frozen brains were cut on a cryostat to get coronal brain sections according to the rat brain atlas. The RVLM punches were made from the frozen brain sections using Stoelting brain punch (Stoelting, IL, USA).

\section{Measurement of RVLM tissue levels of glutamate, GABA and of plasma NE}

RVLM levels of glutamate and GABA were determined by High-performance liquid chromatography with electrochemical detection (ECD, Waters-2465, Waters Corporation, USA) as described previously [27]. Briefly, samples or standards were homogenized in $0.1 \mathrm{~N}$ perchloric acid ( $200 \mu \mathrm{l}$ per sample) and centrifuged at 3, $000 \mathrm{rpm}$ for $3 \mathrm{~min}$. The pellets were washed and resuspended in $1 \mathrm{~N} \mathrm{NaOH}$, and the amount of total protein in each sample was determined by the Bradford method. The separation of glutamate and GABA was achieved with a Novapark $\mathrm{C}_{18}$ reverse-phase column $(150 \times 4.6 \mathrm{~mm}, 4 \mu \mathrm{m}$ particle size, Waters; mobile phase: $100 \mathrm{mM}$ di-sodium hydrogen phosphate anhydrous, $20 \%$ methanol, and $3.5 \%$ acetonitrile at pH 6.8) at $32.5^{\circ} \mathrm{C}$, the flow rate was $1 \mathrm{ml} / \mathrm{min}$ delivered by a Waters pump and then each neurotransmitter was measured by pre-column derivation and ECD. In brief, derivation was achieved by mixing $20 \mu \mathrm{l}$ of working derivation reagent (6.75 mg o-phtaldialdehyde, $2.5 \%$ methanol, $1.25 \mu \mathrm{l} 2$ - $\beta$-mercaptoethanol and $97.5 \%$ $0.1 \mathrm{M}$ sodium tetraborate buffer) with $40 \mu \mathrm{l}$ of filtered supernatant (Nylon-membrane/0.45 lm pore size). The detect channel potentials were set at $+550 \mathrm{mV}$.

The concentration of NE in plasma was determined as described previously [22]. Briefly, plasma samples were collected after rats were decapitated and acidified with glacial acetic acid in $5 \mathrm{ml}$ centrifuge tubes. NE was extracted with $300 \mu \mathrm{l}$ of $0.2 \mathrm{M}$ glacial acetic acid with $10 \mathrm{~min}$ of shaking and a final $40 \mathrm{~min}$ settlement; $20 \mu \mathrm{l}$ of the supernatant was automatically loaded onto a Novapark $\mathrm{C}_{18}$ reverse-phase column $(150 \times 3.2 \mathrm{~mm}, 3 \mu \mathrm{m}$ particle size, Waters $)$ using a refrigerated autoinjector. The mobile phase was composed of $80 \mathrm{mM}$ citric acid monohydrate, $73.4 \mathrm{mM}$ citric acid trisodium salt, $0.12 \mathrm{mM}$ 1-octanesulfonic acid sodium salt, and $0.1 \mathrm{mM}$ EDTA adjust to $\mathrm{pH} 4.3$ with phosphoric acid. The flow rate was set at $0.5 \mathrm{ml} / \mathrm{min}$. Amino acid peaks were identified based on retention time. Extracellular amino acid concentrations were estimated by rationing peak areas of each amino acid and their respective external standard (analytical software: Empower). 


\section{Immunohistochemistry and immunofluorescence studies}

Immunohistochemical labeling was performed in floating sections $(18 \mu \mathrm{m})$ as previously described $[21,22]$ to identify Fra-like (Fra-LI, a marker of chronic neuronal activation), tumour necrosis factor-alpha (TNF- $\alpha$ ) and 67-kDa isoform of glutamate decarboxylase (GAD67) (Santa Cruz Biotechnology, Santa Cruz, California) expressions. Superoxide generation was determined by fluorescent-labelled dihydroethidium (DHE, Molecular Probes) staining as previously described [22, 28]. Protein immunofluorescence staining was performed as previously described $[21,29]$. The primary phosphorylated IKK $\beta$ (p-IKK $\beta$, a marker of NF- $\kappa B$ activation), TNF- $\alpha$ and NAD(P)H oxidase subunit gp91 $91^{\text {phox }}$ antibodies were from Santa Cruz Biotechnology. Conjugated secondary antibodies were used to detect the primary antibody, which included biotinylated secondary antibodies (at 1:300 dilution, ABC staining system kit, Santa Cruz, CA, USA), Alexa 488-labeled anti-rabbit secondary antibody (at 1:200 dilution, green fluorescence), or Alexa 594-labeled anti-mouse secondary antibody (at 1:200 dilution, red fluorescence) (Invitrogen, CA) in 0.1 M PBS (1 hr at room temperature). Immunohistochemistry stained sections were photographed with a conventional light microscopy (DP70, Olympus, Tokyo, Japan). Immunofluorescent staining was visualized with a confocal laser-scanning microscope (Zeiss LSM 710, Carl Zeiss, Inc). For each animal, positive immunofluorescentstaining cells within the RVLM were manually counted in three consecutive sections and an average value was reported.

\section{Western blotting}

Measurement of RVLM protein was performed as previously described [28,30]. Briefly, protein extracted from the RVLM was used for measurements of TNF- $\alpha$, the $67-\mathrm{kDa}$ isoform of glutamate decarboxylase (GAD67), atrial natriuretic peptide (ANP), beta-myosin heavy chain ( $\beta$-MHC), NAD(P)H oxidase subunit NOX 2 (gp91 ${ }^{\text {phox }}$ ) and NOX 4 (Santa Cruz Biotechnology Inc, Santa Cruz, California) expressions by western blot. Protein loading was controlled by probing all blots with $\beta$-actin antibody (Thermo Scientific, USA) and normalizing TNF- $\alpha$, GAD67, ANP, $\beta$-MHC, NOX 2 and NOX4 protein intensities to that of $\beta$-actin. The bands were analyzed using NIH ImageJ software.

\section{ELISA studies}

The levels of TNF- $\alpha$, IL- 6 and IL-1 $\beta$ in plasma and tissues were quantified using commercially available rat ELISA kits (Biosource International Inc, Camarillo, California) according to manufacturer's instructions.

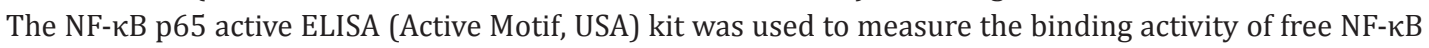
p65 in nuclear extracts, as described previously [21,31].

\section{Statistical analysis}

All data are presented as mean $\pm \mathrm{SE}$ and analyzed using a two-way ANOVA with a Bonferroni posthoc test. Blood pressure data were analyzed by repeated measures ANOVA to examine with-in group changes over time. Statistical comparison was performed using Prism 5 (GraphPad Software). A probability of $P<0.05$ was considered statistically significant.

\section{Results}

\section{Mean arterial pressure (MAP)}

As shown in Fig. 2, high salt diet in sedentary rats caused significant increase in MAP starting at week 8 when compared to NS + Sed and remained increased for the duration of the study. At the end of the study, the results of acute experiment also indicated that HS + Sed rats had higher levels of systolic, diastolic, and mean

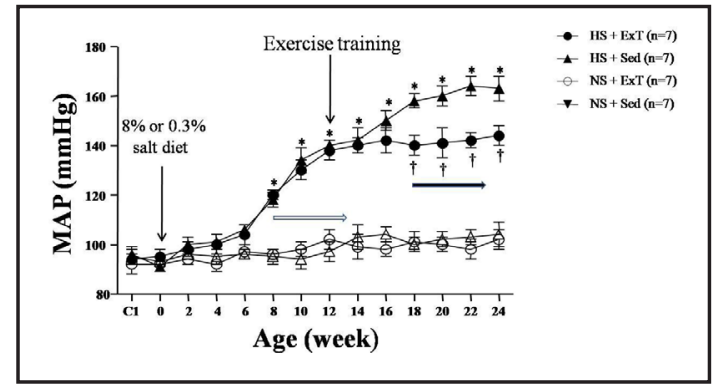

Fig. 2. Time course of mean arterial pressure (MAP) in NS rats and HS rats. MAP was significantly increased in HS + Sed compared with NS + Sed rat from week 8 of high salt diet (empty arrow). MAP was significantly reduced in HS + ExT compared with $\mathrm{HS}+$ Sed rat from week 18 of high salt diet (filled arrow). Values are mean $\pm \mathrm{SE}$; ${ }^{\mathrm{P}}<0.05$ vs. NS groups; $\dagger \mathrm{P}<0.05 \mathrm{HS}+\mathrm{Ex}$ vs. HS + Sed. 
Fig. 3. Effects of exercise training on renal sympathetic nerve activity (RSNA) and the plasma levels of norepinephrine (NE) of NS rats and HS rats. (A) Example of recording RSNA of a rat in each group as indicated. (B) RSNA was increased in HS rats compared with NS rats. Exercise training attenuated RSNA of HS rats. (C) Plasma levels of NE in HS rats were higher than NS rats. Exercise training decreased plasma NE of HS rats. Values are mean $\pm \mathrm{SE} ;{ }^{*} \mathrm{P}<0.05$ vs. NS groups; $+\mathrm{P}<0.05$ HS + Ex vs. HS + Sed.

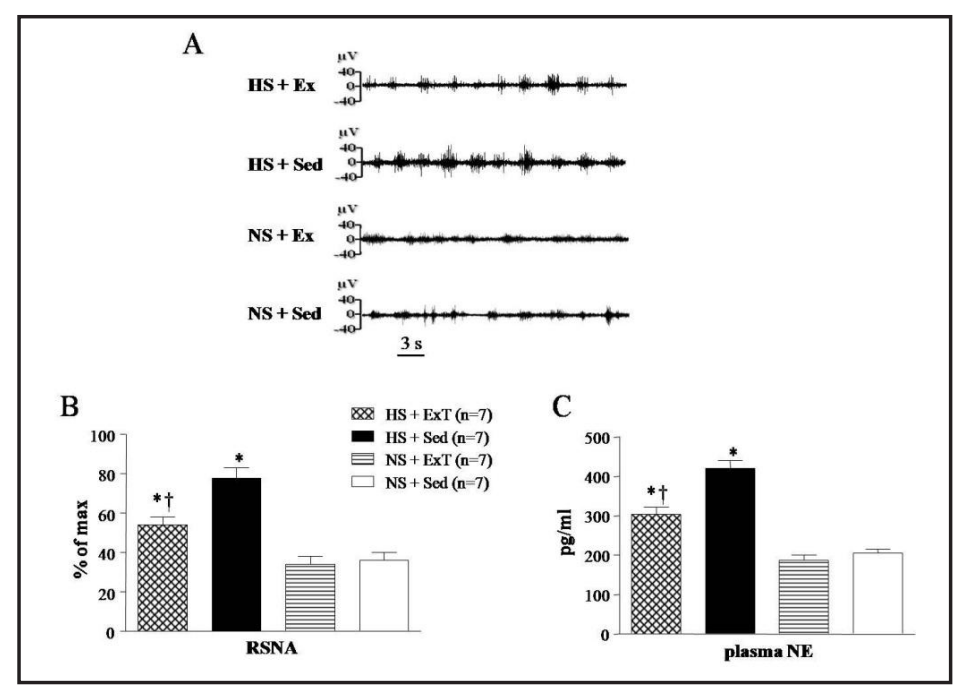

arterial blood pressure (SBP, DBP, and MAP, respectively) when compared to NS + Sed rats (Table 1). Exercise training for 12 weeks prevented high saltinduced increase in MAP, SBP and DBP in comparison with HS + Sed, the MAP was found to be significantly lower in HS + ExT rats from week 18 of exercise training when compared to HS + Sed rats (Fig. 2, Table 1).

\section{Renal sympathetic nerve activity (RSNA)}

Conscious RSNA was measured $5 \mathrm{~h}$ after rats recovered from anesthesia. HS + Sed rats exhibited higher RSNA (\% of max) when compared with NS + Sed rats. Exercise training attenuated RSNA in HS rats (Fig. 3A and B). Plasma NE, a marker of sympathetic activity, was also higher in HS + Sed rats than in NS + Sed rats. Exercise training prevented the increase in plasma NE of HS rats (Fig. 3C).
Table 1. Haemodynamic and anatomical measurements. Data were obtained at conclusion of study. Data are presented as mean \pm SEM. NS, normal salt diet; HS, high salt diet; BW, body weight; HW, heart weight; SBP, systolic blood pressure; DBP, diastolic blood pressure; MAP, mean arterial blood pressure; HR, heart rate. Values are mean \pm SE. $* P<0.05 \mathrm{NS}+$ Ex vs. NS + Sed. $\# P<0.05$ HS + Sed vs. NS groups. $\uparrow P<0.05$ HS + Ex vs. HS + Sed

\begin{tabular}{lcccc}
\hline & NS & NS & HS & HS \\
Parameters & + & + & + & + \\
& Sed & Ex & Sed & Ex \\
\hline $\mathrm{n}$ & 21 & 21 & 21 & 21 \\
Initial BW, g & $292 \pm 2$ & $290 \pm 3$ & $294 \pm 4$ & $293 \pm 2$ \\
Final BW, g & $465 \pm 6$ & $425 \pm 4^{*}$ & $472 \pm 9$ & $418 \pm 4 \dagger$ \\
BW gain, g & $173 \pm 4$ & $135 \pm 3^{*}$ & $178 \pm 6$ & $125 \pm 3 \dagger$ \\
HW, mg & $1851 \pm 18$ & $1901 \pm 20$ & $2451 \pm 26 \#$ & $1889 \pm 20 \dagger$ \\
HW/BW, mg/g & $3.98 \pm 0.03$ & $4.47 \pm 0.05^{*}$ & $5.19 \pm 0.1 \#$ & $4.51 \pm 0.07 \dagger$ \\
SBP, mmHg & $146 \pm 15$ & $142 \pm 13$ & $188 \pm 18 \#$ & $172 \pm 17 \# \dagger$ \\
DBP, mmHg & $88 \pm 11$ & $84 \pm 10$ & $152 \pm 15 \#$ & $113 \pm 10 \# \dagger$ \\
MAP, mmHg & $107 \pm 3$ & $103 \pm 2$ & $164 \pm 14 \#$ & $132 \pm 13 \# \dagger$ \\
HR, bpm & $372 \pm 12$ & $330 \pm 9^{*}$ & $412 \pm 10 \#$ & $366 \pm 8 \dagger$ \\
\hline
\end{tabular}

\section{TNF- $\alpha$ expression and NF- $\kappa B$ activation in the RVLM}

$\mathrm{HS}+$ Sed rats showed increases in the level of TNF- $\alpha$ (Fig. 4A and C), p-IKK $\beta$ (Fig. 4B and D) and NF-кB p65 activity (Fig. 4E) in the RVLM compared with NS + Sed rats. Exercise training attenuated the increases in RVLM TNF- $\alpha$ expression, NF- $\kappa B$ p65 activity and p-IKK $\beta$ in HS rats (Fig. 4).

\section{Fra-LI and GAD67 expression in the RVLM}

To determine whether exercise training influences sympathoexcitation in the RVLM, we examined the expression of Fra-like (Fra-LI, fos family gene; indicating chronic neuronal excitation) by immunohistochemistry staining. To further determine whether exercise training induced effects are mediated by alterations in neurotransmitter in the RVLM, we determined the level of 67-kDa isoform of glutamate decarboxylase (GAD67) in the RVLM. 
Fig. 4. Effects of exercise training on TNF- $\alpha$ expression and NF- $\kappa \mathrm{B}$ activation in the RVLM of NSrats and HS rats. (A and C) A representative immunohistochemistry image and the column diagram showing the effects of exercise training on the positive neurons of TNF- $\alpha$ in the RVLM of NS rats and HS rats. ( $\mathrm{B}$ and $\mathrm{D}$ ) A representative immunofluorescence image and the column diagram showing the effects of exercise training on the positive neurons of $\mathrm{p}$-IKK $\beta$ in the

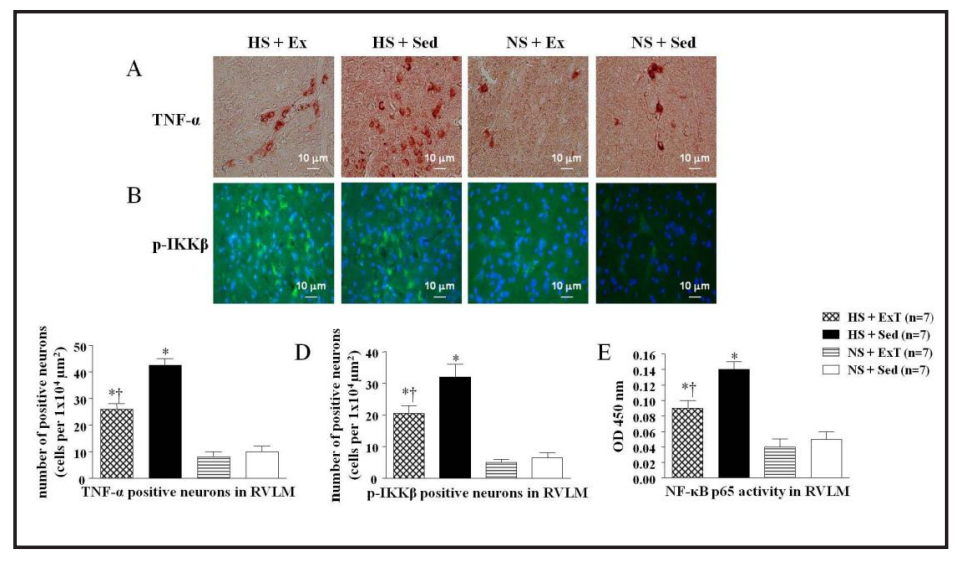
RVLM of NS rats and HS rats. (E) NF- $\kappa$ B activity assay showing increased NF- $\kappa B$ p65 activity in RVLM of HS + Sed rats when compared to NS + Sed rats, whereas, exercise training attenuated the increases in RVLM NF- $\kappa B$ p 65 activity in HS rats. Values are mean $\pm S E$. $* P<0.05$ vs. NS groups; $\uparrow \mathrm{P}<0.05$ HS + Ex vs. HS + Sed.

Fig. 5. Effects of exercise training on the $67-\mathrm{kDa}$ isoform of glutamate decarboxylase (GAD67) immunoreactivity and neuronal activity in the RVLM of NS rats and HS rats. (A and C) A representative immunohistochemistry image and the column diagram showing the effects of exercise training on the positive neurons of GAD67 (brown yellow) in the RVLM of NS rats and HS rats. (B and D) A representative immunohistochemistry image and the column diagram showing the effects of exercise training on the Fra-LI (brown dots, an indicator of chronic neuronal excitation)
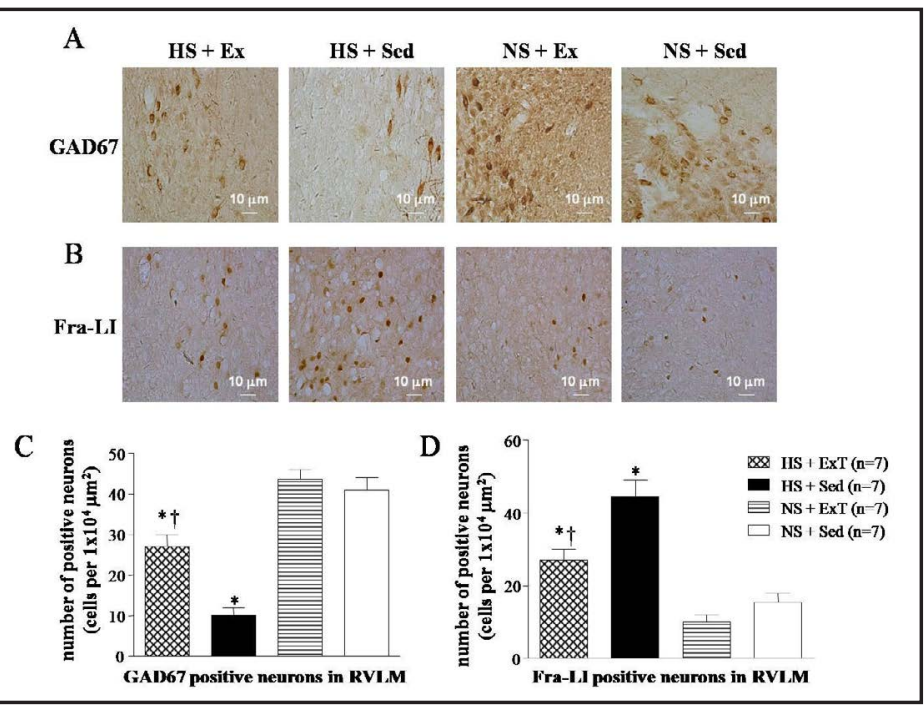
expression in the RVLM of NS rats and HS rats. Values are mean $\pm \mathrm{SE}$. ${ }^{*} \mathrm{P}<0.05$ vs. NS groups; $\uparrow \mathrm{P}<0.05 \mathrm{HS}+\mathrm{Ex}$ vs. HS + Sed.

We observed that HS + Sed rats exhibited lower level of GAD67 in the RVLM compared to $\mathrm{NS}+$ Sed rats. Interestingly, exercise training significantly upregulated GAD67 level in HS rats compared to NS rats (Fig. 5A and C).

Furthermore, we found that HS + Sed rats exhibited increased Fra-LI activity in the RVLM neurons compared to NS + Sed rats. Notably, this upregulation of Fra-LI activity was significantly attenuated by exercise training in HS rats. However, exercise training did not change Fra-LI activity in NS rats (Fig. $5 B$ and D).
Table 2. The RVLM and plasma levels of pro-inflammatory cytokines ( $\mathrm{n}=$ 7). Values are mean \pm SE. $* P<0.05$ HS + Sed vs. NS groups. $\uparrow P<0.05$ HS + Ex vs. HS + Sed

\begin{tabular}{lllllll}
\hline Group & \multicolumn{4}{l}{ RVLM $(\mathrm{pg} / \mathrm{mg}$ protein, $\mathrm{n}=7)$} & \multicolumn{2}{l}{ Plasma $(\mathrm{pg} / \mathrm{mL}, \mathrm{n}=7)$} \\
\hline & TNF- $\alpha$ & IL-1 $\beta$ & IL-6 & TNF- $\alpha$ & IL-1 $\beta$ & IL-6 \\
NS + Ex & $2.1 \pm 0.2$ & $17.4 \pm 1.8$ & $17.9 \pm 1.7$ & $11.2 \pm 1.5$ & $58.3 \pm 5.4$ & $40.2 \pm 2.9$ \\
NS + Sed & $3.0 \pm 0.3$ & $19.1 \pm 2.2$ & $22.5 \pm 2.1$ & $12.4 \pm 1.5$ & $60.5 \pm 5.7$ & $49.6 \pm 3.7$ \\
HS + Ex & $4.2 \pm 0.4^{* \dagger}$ & $34.6 \pm 3.2^{*} \dagger$ & $39.6 \pm 4.2^{*} \dagger$ & $13.9 \pm 1.8 \dagger$ & $91.6 \pm 8.9^{*} \dagger$ & $85.7 \pm 8.2^{*} \dagger$ \\
HS + Sed & $6.2 \pm 0.7^{*}$ & $55.3 \pm 4.8^{*}$ & $69.7 \pm 6.6^{*}$ & $28.1 \pm 3.2^{*}$ & $125.6 \pm 12.4^{*}$ & $114.6 \pm 10.2^{*}$ \\
\hline
\end{tabular}


PICs in the RVLM and plasma

To investigate the influence of exercise training on PICs within the RVLM and plasma of hypertensive rats, we examined the levels of TNF- $\alpha$, IL- 6 and IL- $1 \beta$ in the RVLM and plasma. We observed that the levels of TNF- $\alpha$, IL- 6 and IL- $1 \beta$ exhibited marked increases in the RVLM and plasma in HS rats compared to NS rats (Table 2). This upregulation of TNF- $\alpha$, IL-6 and IL-1 $\beta$ was significantly attenuated by exercise training in HS rats (Table 2 ).

\section{Superoxide and $N A D(P) H$ oxidase in the RVLM}

HS + Sed rats had more superoxide in the RVLM, as determined by fluorescent labeled dihydroethidium (DHE) and the NAD(P)H oxidase subunit NOX 2 (gp91 ${ }^{\text {phox }}$ ) and NOX 4, when compared with NS + Sed rats (Fig. 6). Exercise training decreased gp91 ${ }^{\text {phox }}$, NOX 4 and DHE in the RVLM of HS rats (Fig. 6).

Fig. 6. Superoxide and $\mathrm{NAD}(\mathrm{P}) \mathrm{H}$ oxidase in the RVLM of NS rats and HS rats. (A) Immunofluorescence for the NAD(P)H oxidase subunit gp91 $1^{\text {phox }}$ (red) and superoxide as determined by fluorescentlabeled dihydroethidium (DHE) in the RVLM in different groups. (B) Comparison of gp91 $91^{\text {phox }}$ positive neurons in the RVLM in different groups. (C) Immunofluorescent intensity of DHE in the RVLM of different groups of rats. Values are mean $\pm \mathrm{SE}$. ${ }^{*} \mathrm{P}<0.05$ vs. NS groups; $\dagger \mathrm{P}<0.05$ HS + Ex vs. HS + Sed.

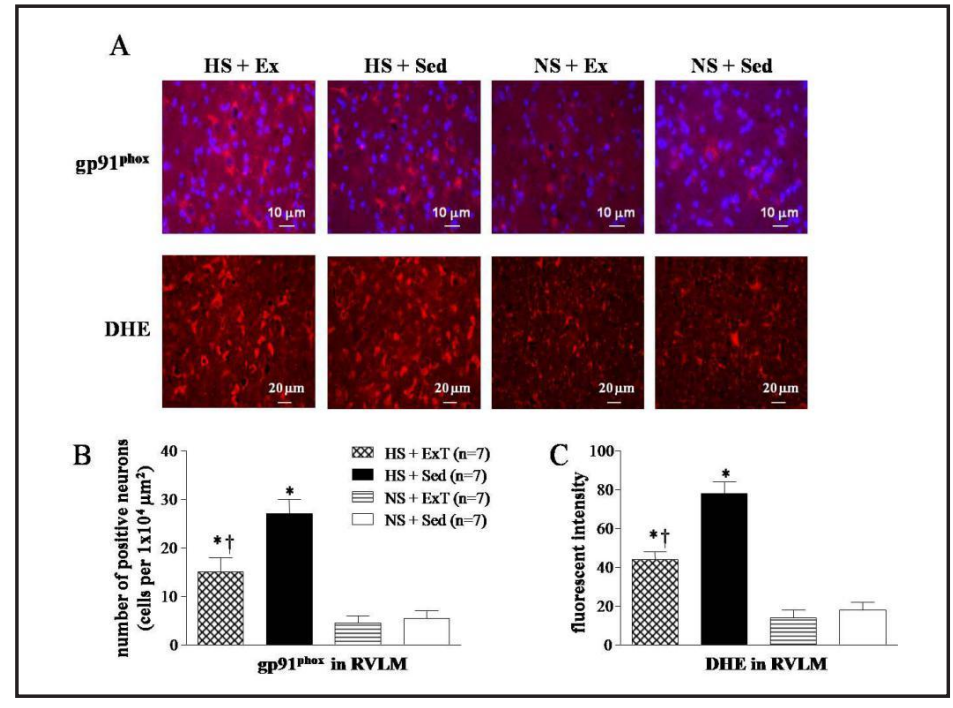

Fig. 7. Protein expression of TNF- $\alpha$, GAD67, gp91 ${ }^{\text {phox }}$ and NOX4 in the RVLM and ANP and $\beta$-MHC in the heart of NS rats and HS rats. (A) HS rats had higher levels of TNF- $\alpha$ and lower levels of GAD67 in the RVLM when compared with NS rats. Exercise training decreased the protein expression of TNF- $\alpha$ and increased the protein express of GAD67 in the RVLM of HS rats. (B) HS rats had higher levels of gp91 $1^{\text {phox }}$ and NOX4 in the RVLM when compared with NS rats. Exercise training decreased the protein expression of gp $91^{\text {phox }}$ and NOX4 in the RVLM when compared

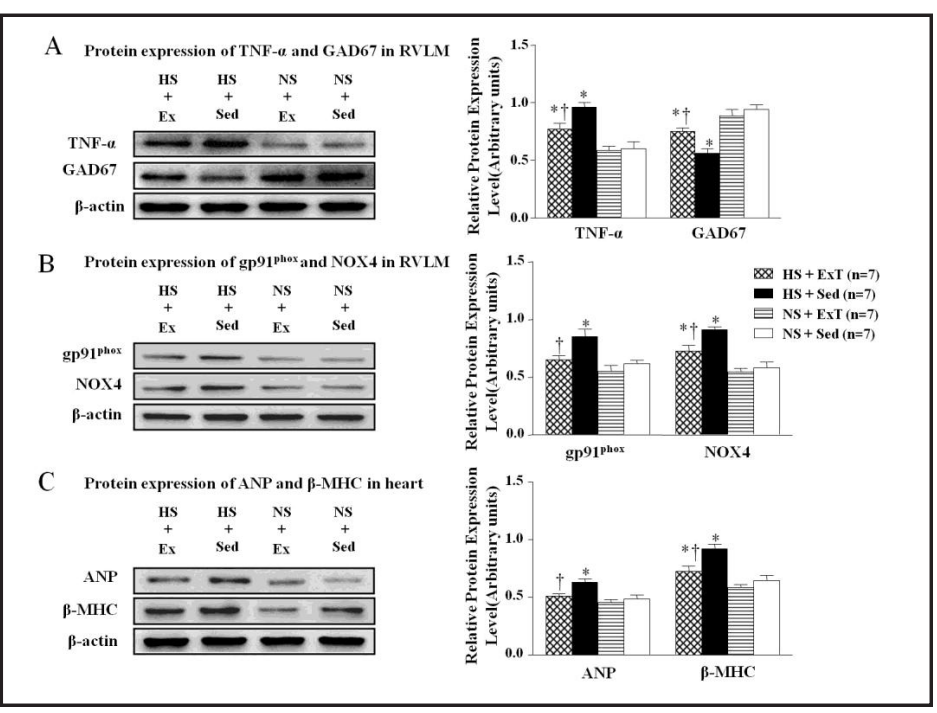
with NS rats. (C) Western blot for ANP and $\beta$-MHC expressions in the heart were lower in exercise training-treated HS rats than in sedentary HS rats. Values are mean $\pm \mathrm{SE}$. $* \mathrm{P}<0.05$ vs. NS groups; $\uparrow \mathrm{P}<0.05$ HS + Ex vs. HS + Sed. 
Fig. 8. Laser confocal images $(\times 40)$ showing co-expression of TNF- $\alpha$ (red) and gp91 ${ }^{\text {phox }}$ (green) in the RVLM of NS rats and HS rats. (A) Representative images of co-expression of TNF- $\alpha$ (red) and gp91 $1^{\text {phox }}$ (green) in the RVLM of NS rats and HS rats. (B) Corresponding quantification ( $\mathrm{n}=5$ per group). Values are mean $\pm \mathrm{SE}$. $* \mathrm{P}<0.05$ vs. NS groups; $\uparrow \mathrm{P}<0.05$ HS + Ex vs. HS + Sed.

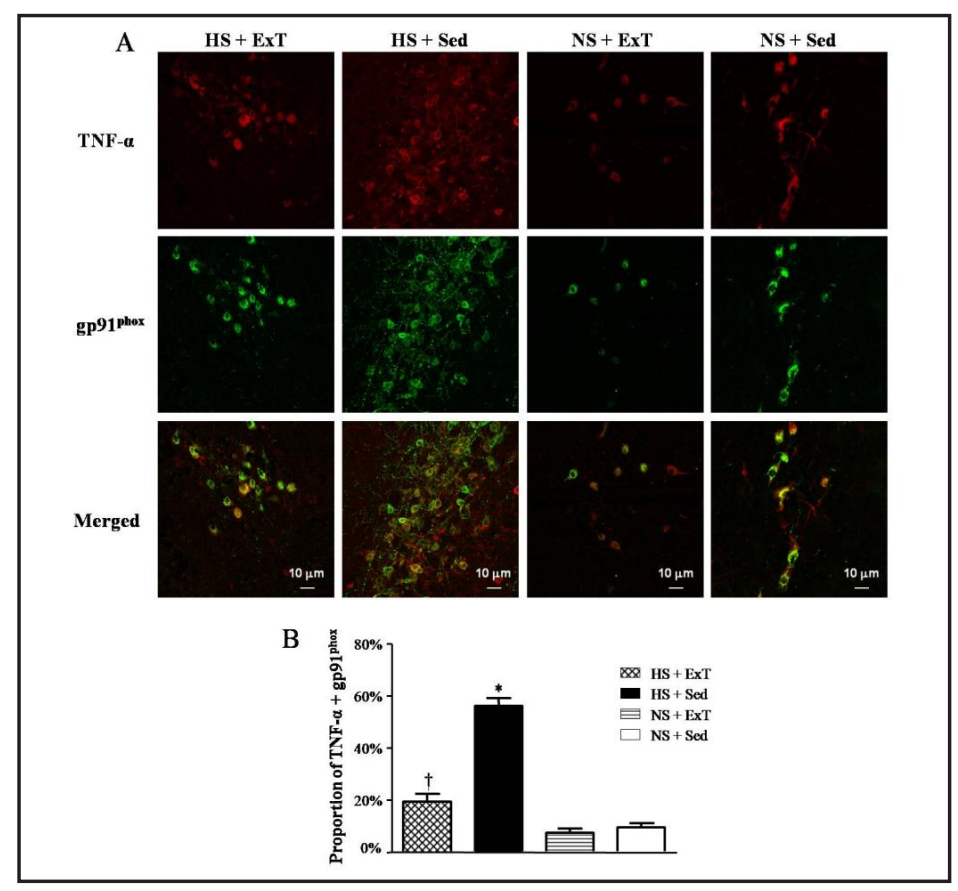

The protein expression of TNF- $\alpha$, GAD67, gp91 $1^{\text {phox }}$ and NOX 4 in the RVLM

HS + Sed rats had higher levels of TNF- $\alpha$, gp $91^{\text {phox }}$ and NOX 4, and lower level of GAD67 in the RVLM than those of $\mathrm{NS}+$ Sed rats (Fig. 7A, B). Exercise training attenuated the increases TNF- $\alpha$, gp $91^{\text {phox }}$ and NOX 4, and a decrease in GAD67 in the RVLM of HS rats (Fig. 7A, B).
Table 3. Neurotransmitters in the RVLM $(n=7)$. Values are mean \pm SE. $* P<0.05$ HS + Sed vs. NS groups. $\uparrow P<0.05$ HS + Ex vs. HS + Sed

\begin{tabular}{lllll}
\hline Group & NS + Ex & NS + Sed & HS + Ex & HS + Sed \\
\hline glutamate (ng/mg) & $287 \pm 52$ & $301 \pm 58$ & $391 \pm 72 * \dagger$ & $490 \pm 80^{*}$ \\
GABA (ng/mg) & $297 \pm 49$ & $314 \pm 52$ & $205 \pm 48 * \dagger$ & $128 \pm 43 *$ \\
\hline
\end{tabular}

\section{Cardiac hypertrophy}

The heart weight/body weight (HW/BW) ratio was measured as indicators of cardiac hypertrophy. HS rats had increased cardiac hypertrophy, as assessed by the ratio of heart weight to body weight (HW/BW) (Table 1), which were reduced following exercise training. The protein expressions of markers of cardiac hypertrophy, atrial natriuretic peptide (ANP) and beta-myosin heavy chain ( $\beta$-MHC) were measured in the left ventricular tissue of the heart using western blot. HS rats resulted in increased protein expression of ANP and $\beta$-MHC in the left ventricular tissue of the heart, which were decreased by exercise training (Fig. 7C).

\section{Neurotransmitters in the RVLM}

HS rats had elevated level of glutamate and lower level of GABA in the RVLM. Exercise training prevented the decrease in RVLM GABA and the increase in RVLM glutamate in HS rats (Table 3 ).

\section{Co-expression of TNF- $\alpha$ and gp $91^{\text {phox }}$ in the RVLM}

To check the co-localization of TNF- $\alpha$-positive neurons and gp91 ${ }^{\text {phox }}$-positive neurons in the RVLM, we performed double labeling studies. Double labeling results also revealed that $56.2 \%$ of the TNF- $\alpha$-positive neurons are also positive for gp9 $91^{\text {phox }}$ in HS rats (Fig. 8). Only $19.8 \%$ of TNF- $\alpha$-positive neurons were positive for gp $91^{\text {phox }}$ in the RVLM of HS rats following exercise training (Fig. 8). 


\section{Discussion}

In the present study, we evaluated the impact of exercise training of 12 weeks on blood pressure and cardiac hypertrophy in salt-induced hypertension. The novel findings of this study are that: (1) exercise training may attenuate hypertension induced cardiac hypertrophy and sympathoexcitation by restoring the balances between the excitatory and inhibitory neurotransmitters in RVLM; (2) NF- $\kappa B$ activity, oxidative stress and PIC in the RVLM may be involved in the exercise training induced effects.

Exercise training (ExT), a part of lifestyle modification, has been recognized as an important strategy for antihypertension $[9,20]$. In this work, we observed significant reduction in MAP in trained hypertensive rats compared with their sedentary counterparts and saw no comparable changes in trained normotensive controls. As shown in Fig. 2, high salt diet resulted in significant increase in MAP in sedentary rats beginning from week 8 and remained increased for the duration of the study. Regular exercise training resulted in significant reduction in MAP beginning from week 18 of training and remained significantly lower until the end of the study. Our study also showed that exercise training resulted in reduced cardiac hypertrophy in HS rats, as indicated by decreased whole heart weight/ bodyweight ratio, protein expressions of atrial natriuretic peptide and beta-myosin heavy chain. These results of the present study suggest that regular exercise delays the progression of hypertension. This finding is significant from a clinical perspective, because evidence suggest that a reduction of BP by only $5 \mathrm{mmHg}$ can significantly reduces the risk of stroke, heart failure, and mortality from cardiovascular diseases [32].

Although the peripheral mechanisms underlying the cardiovascular changes evoked by exercise training have been widely described [11-13], the central mechanisms involved in cardiovascular control that can be changed by ExT are not well understood. It is well known that the RVLM is a key region for central control of sympathetic outflow and plays a crucial role in maintaining resting BP and sympathetic tone [33, 34]. The tonic activity of the RVLM is related to the interplay of a number of neurotransmitters and neuromodulators in these sympathetic neurons. The sympathetic outflow from the RVLM depends upon the balance of these excitatory and inhibitory neuromodulators, including glutamate, GABA and angiotensin II $[19,35]$. In this study, we observed that HS rats exhibited an increase in the circulating plasma NE (an indirect marker of sympathetic activity) and elevated RSNA, and increased the level of glutamate as well as increased the expression of Fra-LI activity in RVLM neurons (indicative of increased neuronal activity) when compared to NS rats. Concomitantly, when compared to NS rats, HS rats had significantly reduced the levels of GABA and GAD67, a marker to recognize GABAergic neurons in the RVLM. More importantly, exercise training attenuated sympathetic excitation, caused reduction in Fra-LI staining and prevented the increase in glutamate and decreases in GABA and GAD67 in the RVLM of HS rats. These results provide sufficient evidence that exercise training may restore the balance between excitatory and inhibitory neurotransmitter in the RVLM and play an important role in the pathogenesis of salt-induced hypertension.

Increased proinflammatory cytokines (PIC) expression in autonomic control areas, as the RVLM, has been identified as a major central molecular mechanism to decrease increase pressure variability and renal sympathetic nerve activity, thus contributing to the development/maintenance of hypertension $[5,36]$. Nuclear factor-kappa B (NF- $\kappa B$ ) is one of the most important downstream transcription factors responsible for the transcription of PIC, and thereby regulates BP [20,21]. It has been demonstrated that increased PIC activate NF- $\kappa B$ signaling pathway and activation of NF- $\kappa B$ in turn further upregulates PIC [20]. A grow body of evidence has shown that ExT decreases the pro-inflammatory profile in autonomic brain areas, increases baroreflex function, and attenuates sympathetic activity in angiotensin II infused rats and heart failure rabbits $[16,17,37,38]$. In the spontaneously hypertensive rats it was also shown that sinoaortic denervation blocked ExT-induced resting bradycardia, pressure fall and reduced sympathetic vasomotor variability $[10,39$, 40]. In this study, we also observed that exercise training reduced PIC expression, p-IKK $\beta$ 
and NF- $\kappa B$ p65 activity in the RVLM of HS rats, but not in NS rats. Meanwhile, the significant reductions in circulating plasma levels of PIC (TNF- $\alpha$, IL-1 $\beta$, and IL-6) and NE in HS + ExT rats were observed when compared to HS + Sed rats. There are no comparable changes in normotensive rats receiving ExT. These results suggest that some of the beneficial effects of ExT in salt-induced hypertension are mediated by attenuated the level of PIC in the RVLM.

Previous studies have shown that oxidative stress, particularly the superoxide anion $\left(\mathrm{O}_{2}{ }^{--}\right)$, plays a key role in the development of hypertension and cardiac hypertrophy. NAD(P) $\mathrm{H}$ oxidase, a complex enzyme consisting of two membrane-bound components (gp91 ${ }^{\text {phox }}$ and $\mathrm{p} 22^{\text {phox }}$ ) and three cytosolic components (p67 phox, $\mathrm{p} 47^{\text {phox }}$, and p40 ${ }^{\text {phox }}$ [41], can be activated by PIC and NF- $\kappa B$ in peripheral tissues. Interestingly, cytoplasmic and membrane-associated $\mathrm{NAD}(\mathrm{P}) \mathrm{H}$ oxidase proteins have been found throughout the neuraxis, indicating that NAD(P) $\mathrm{H}$ oxidase-dependent oxidative stress are also generated within the nervous system . Our recent studies also indicate that cytokines and their transcription factor, NF- $\kappa \mathrm{B}$, contribute to the induction of NADPH oxidase (NOX) derived reactive oxygen species (ROS) in heart failure [42] and hypertension [31]. There is growing evidence from studies on animals that exercise training prevents cellular damage by reducing oxidative stress in hypertension [20, $43,44]$. One of the mechanisms underlying the effect of exercise training on oxidative damage could be decreased protein expression of NAD(P)H oxidases and/or inactivation of these proteins, as evidenced in various animal models of hypertension $[41,45]$. In the present study, we also found that exercise training resulted in attenuation of increased expression of the NAD(P)H oxidase subunit gp91 ${ }^{\text {phox }}$ and ROS (DHE staining) in the RVLM of HS rats. More importantly, exercise training resulted in downregulation of NF- $\kappa B$ activity in HS rats. Therefore, one possible mechanism by which exercise training exerts its beneficial effects could be via downregulation of NF- $\kappa$ B activity, thereby attenuating oxidative stress in the RVLM of salt-induced hypertension.

Given the results from that report and our current results, it is plausible to suggest that ExT may be contributed to reductions of PIC and ROS production and of an imbalance between excitatory and inhibitory neurotransmitters within the RVLM, and thus, lead to disruption of the positive feedback cycle involved with hypertension and cardiac hypertrophy. Our findings provide further evidence and insight for the beneficial effect of exercise training on hypertension and cardiac hypertrophy.

\section{Acknowledgements}

This work was supported by National Natural Science Foundation of China (Nos. 81770426, 81700373 and 91639105), China Postdoctoral Science Foundation (grant numbers 2016M592802), Natural Science Foundation of Shaanxi (Nos. 2017JQ8011) and Shaanxi Postdoctoral Science Foundation (grant numbers 2016BSHEDZZ88).

\section{Disclosure Statement}

The authors declare to have no conflict of interests.

\section{References}

1 Dange RB, Agarwal D, Masson GS, Vila J, Wilson B, Nair A, Francis J: Central blockade of TLR4 improves cardiac function and attenuates myocardial inflammation in angiotensin II-induced hypertension. Cardiovasc Res 2014;103:17-27.

2 Guyenet PG: The sympathetic control of blood pressure. Nat Rev Neurosci 2006;7:335-346.

-3 Raquel Hde A, Masson GS, Barna BF, Zanluqui NG, Pinge-Filho P, Michelini LC, Martins-Pinge MC: Swimming Training Modulates Nitric Oxide-Glutamate Interaction in the Rostral Ventrolateral Medulla in Normotensive Conscious Rats. Front Physiol 2016;7:221. 


\section{Cellular Physiology Cell Physiol Biochem 2018;48:1369-1381 \begin{tabular}{ll|l} 
and Biochemistry Published onIIne: July 26, 2018 & $\begin{array}{l}\text { C) } 2018 \text { The Author(s). Published by S. Karger AG, Basel } \\
\text { www.karger.com/cpb }\end{array}$ \\
\hline
\end{tabular}}

-4 Wang YK, Shen D, Hao Q Yu Q, Wu ZT, Deng Y, Chen YF, Yuan WJ, Hu QK, Su DF, Wang WZ: Overexpression of angiotensin-converting enzyme 2 attenuates tonically active glutamatergic input to the rostral ventrolateral medulla in hypertensive rats. Am J Physiol Heart Circ Physiol 2014;307:H182-H190.

5 Wu KL, Chan SH, Chan JY: Neuroinflammation and oxidative stress in rostral ventrolateral medulla contribute to neurogenic hypertension induced by systemic inflammation. J Neuroinflammation 2012;9:212.

6 Sousa LE, Magalhaes WG, Bezerra FS, Santos RA, Campagnole-Santos MJ, Isoldi MC, Alzamora AC: Exercise training restores oxidative stress and nitric oxide synthases in the rostral ventrolateral medulla of renovascular hypertensive rats. Free Radic Res 2015;49:1335-1343.

7 Agarwal D, Elks CM, Reed SD, Mariappan N, Majid DS, Francis J: Chronic exercise preserves renal structure and hemodynamics in spontaneously hypertensive rats. Antioxid Redox Signal 2012;16:139-152.

8 Nascimento LS, Santos AC, Lucena J, Silva L, Almeida A, Brasileiro-Santos MS: Acute and chronic effects of aerobic exercise on blood pressure in resistant hypertension: study protocol for a randomized controlled trial. Trials 2017;18:250.

-9 Masson GS, Nair AR, Silva Soares PP, Michelini LC, Francis J: Aerobic training normalizes autonomic dysfunction, HMGB1 content, microglia activation and inflammation in hypothalamic paraventricular nucleus of SHR. Am J Physiol Heart Circ Physiol 2015;309:H1115-H1122.

10 Chaar LJ, Alves TP, Batista Junior AM, Michelini LC: Early Training-Induced Reduction of Angiotensinogen in Autonomic Areas-The Main Effect of Exercise on Brain Renin-Angiotensin System in Hypertensive Rats. PLoS One 2015;10:e0137395.

11 Muller-Delp JM, Hotta K, Chen B, Behnke BJ, Maraj JJ, Delp MD, Lucero TR, Bramy JA, Alarcon DB, Morgan HE, Cowan MR, Haynes AD: Effects of age and exercise training on coronary microvascular smooth muscle phenotype and function. J Appl Physiol (1985) 2018;124: 140-149.

12 Trinity JD, Broxterman RM, Richardson RS: Regulation of exercise blood flow: Role of free radicals. Free Radic Biol Med 2016;98:90-102.

13 Higashi Y, Yoshizumi M: Exercise and endothelial function: role of endothelium-derived nitric oxide and oxidative stress in healthy subjects and hypertensive patients. Pharmacol Ther 2004;102:87-96.

14 Laterza MC, de Matos LD, Trombetta IC, Braga AM, Roveda F, Alves MJ, Krieger EM, Negrao CE, Rondon MU: Exercise training restores baroreflex sensitivity in never-treated hypertensive patients. Hypertension 2007;49:1298-1306.

15 Ren CZ, Yang YH, Sun JC, Wu ZT, Zhang RW, Shen D, Wang YK: Exercise Training Improves the Altered Renin-Angiotensin System in the Rostral Ventrolateral Medulla of Hypertensive Rats. Oxid Med Cell Longev 2016;2016:7413963.

16 Andrade DC, Arce-Alvarez A, Toledo C, Diaz HS, Lucero C, Schultz HD, Marcus NJ, Del Rio R: Exercise training improves cardiac autonomic control, cardiac function, and arrhythmogenesis in rats with preserved-ejection fraction heart failure. J Appl Physiol (1985) 2017;123:567-577.

17 Ichige MH, Santos CR, Jordao CP, Ceroni A, Negrao CE, Michelini LC: Exercise training preserves vagal preganglionic neurones and restores parasympathetic tonus in heart failure. J Physiol 2016;594:62416254.

18 Zha YP, Wang YK, Deng Y, Zhang RW, Tan X, Yuan WJ, Deng XM, Wang WZ: Exercise training lowers the enhanced tonically active glutamatergic input to the rostral ventrolateral medulla in hypertensive rats. CNS Neurosci Ther 2013;19:244-251.

19 Agarwal D, Welsch MA, Keller JN, Francis J: Chronic exercise modulates RAS components and improves balance between pro- and anti-inflammatory cytokines in the brain of SHR. Basic Res Cardiol 2011;106:1069-1085.

20 Agarwal D, Haque M, Sriramula S, Mariappan N, Pariaut R, Francis J: Role of proinflammatory cytokines and redox homeostasis in exercise-induced delayed progression of hypertension in spontaneously hypertensive rats. Hypertension 2009;54:1393-1400.

21 Li HB, Li X, Huo CJ, Su Q, Guo J, Yuan ZY, Zhu GQ, Shi XL, Liu JJ, Kang YM: TLR4/MyD88/NF-kappaB signaling and PPAR-gamma within the paraventricular nucleus are involved in the effects of telmisartan in hypertension. Toxicol Appl Pharmacol 2016;305:93-102.

22 Li HB, Qin DN, Cheng K, Su Q, Miao YW, Guo J, Zhang M, Zhu GQ Kang YM: Central blockade of salusin beta attenuates hypertension and hypothalamic inflammation in spontaneously hypertensive rats. Sci Rep 2015;5:11162.

23 Llewellyn T, Zheng H, Liu X, Xu B, Patel KP: Median preoptic nucleus and subfornical organ drive renal sympathetic nerve activity via a glutamatergic mechanism within the paraventricular nucleus. Am J Physiol Regul Integr Comp Physiol 2012;302:R424-R432.

24 Kang YM, Wang Y, Yang LM, Elks C, Cardinale J, Yu XJ, Zhao XF, Zhang J, Zhang LH, Yang ZM, Francis J: TNFalpha in hypothalamic paraventricular nucleus contributes to sympathoexcitation in heart failure by 


\section{Cellular Physiology Cell Physiol Biochem 2018;48:1369-1381 \begin{tabular}{l|l} 
and Biochemistry Published onlıne: July 26, 2018 & $\begin{array}{l}\text { (c) } 2018 \text { The Author(s). Published by S. Karger AG, Basel } \\
\text { www.karger.com/cpb }\end{array}$
\end{tabular}}

Li et al.: Exercise Training in Salt-Induced Hypertension

modulating AT1 receptor and neurotransmitters. Tohoku J Exp Med 2010;222:251-263.

25 Liu JL, Irvine S, Reid IA, Patel KP, Zucker IH: Chronic exercise reduces sympathetic nerve activity in rabbits with pacing-induced heart failure: A role for angiotensin II. Circulation 2000;102:1854-1862.

26 Kent BB, Drane JW, Blumenstein B, Manning JW: A mathematical model to assess changes in the baroreceptor reflex. Cardiology 1972;57:295-310.

-27 Zhang B, Chu J, Zhang J, Ma Y: Change of extracellular glutamate and gamma-aminobutyric acid in substantia nigra and globus pallidus during electrical stimulation of subthalamic nucleus in epileptic rats. Stereotact Funct Neurosurg 2008;86:208-215.

-28 Wang YK, Yu Q, Tan X, Wu ZT, Zhang RW, Yang YH, Yuan WJ, Hu QK, Wang WZ: Centrally acting drug moxonidine decreases reactive oxygen species via inactivation of the phosphoinositide-3 kinase signaling in the rostral ventrolateral medulla in hypertensive rats. J Hypertens 2016;34:993-1004.

-29 Yang YH, Li DL, Bi XY, Sun L, Yu XJ, Fang HL, Miao Y, Zhao M, He X, Liu JJ, Zang WJ: Acetylcholine Inhibits LPS-Induced MMP-9 Production and Cell Migration via the alpha7 nAChR-JAK2/STAT3 Pathway in RAW264.7 Cells. Cell Physiol Biochem 2015;36:2025-2038.

30 Dai P, Mao Y, Sun X, Li X, Muhammad I, Gu W, Zhang D, Zhou Y, Ma J, Ni Z, Huang S: Attenuation of Oxidative Stress-Induced Osteoblast Apoptosis by Curcumin is Associated with Preservation of Mitochondrial Functions and Increased Akt-GSK3beta Signaling. Cell Physiol Biochem 2017;41:661-677.

31 Kang YM, Ma Y, Zheng JP, Elks C, Sriramula S, Yang ZM, Francis J: Brain nuclear factor-kappa B activation contributes to neurohumoral excitation in angiotensin II-induced hypertension. Cardiovasc Res 2009;82:503-512.

-32 Law M, Wald N, Morris J: Lowering blood pressure to prevent myocardial infarction and stroke: a new preventive strategy. Health Technol Assess 2003;7:1-94.

33 Du D, Hu L, Wu J, Wu Q Cheng W, Guo Y, Guan R, Wang Y, Chen X, Yan X, Zhu D, Wang J, Zhang S, Xia C: Neuroinflammation contributes to autophagy flux blockage in the neurons of rostral ventrolateral medulla in stress-induced hypertension rats. J Neuroinflammation 2017;14:169.

34 Li HB, Qin DN, Ma L, Miao YW, Zhang DM, Lu Y, Song XA, Zhu GQ Kang YM: Chronic infusion of lisinopril into hypothalamic paraventricular nucleus modulates cytokines and attenuates oxidative stress in rostral ventrolateral medulla in hypertension. Toxicol Appl Pharmacol 2014;279:141-149.

-35 Gabor A, Leenen FH: Central neuromodulatory pathways regulating sympathetic activity in hypertension. J Appl Physiol (1985) 2012;113:1294-1303.

-36 Shi P, Grobe JL, Desland FA, Zhou G, Shen XZ, Shan Z, Liu M, Raizada MK, Sumners C: Direct proinflammatory effects of prorenin on microglia. PLoS One 2014;9:e92937.

-37 Pan YX, Gao L, Wang WZ, Zheng H, Liu D, Patel KP, Zucker IH, Wang W: Exercise training prevents arterial baroreflex dysfunction in rats treated with central angiotensin II. Hypertension 2007;49:519-527.

38 Gao L, Wang W, Liu D, Zucker IH: Exercise training normalizes sympathetic outflow by central antioxidant mechanisms in rabbits with pacing-induced chronic heart failure. Circulation 2007;115:3095-3102.

-39 Ceroni A, Chaar LJ, Bombein RL, Michelini LC: Chronic absence of baroreceptor inputs prevents traininginduced cardiovascular adjustments in normotensive and spontaneously hypertensive rats. Exp Physiol 2009;94:630-640.

40 Ogihara CA, Schoorlemmer GH, Lazari Mde F, Giannocco G, Lopes OU, Colombari E, Sato MA: Swimming exercise changes hemodynamic responses evoked by blockade of excitatory amino receptors in the rostral ventrolateral medulla in spontaneously hypertensive rats. Biomed Res Int 2014;2014:487129.

41 Zalba G, Beaumont FJ, San Jose G, Fortuno A, Fortuno MA, Etayo JC, Diez J: Vascular NADH/NADPH oxidase is involved in enhanced superoxide production in spontaneously hypertensive rats. Hypertension 2000;35:1055-1061.

42 Kang YM, Ma Y, Elks C, Zheng JP, Yang ZM, Francis J: Cross-talk between cytokines and renin-angiotensin in hypothalamic paraventricular nucleus in heart failure: role of nuclear factor-kappaB. Cardiovasc Res 2008;79:671-678.

43 Roque FR, Briones AM, Garcia-Redondo AB, Galan M, Martinez-Revelles S, Avendano MS, Cachofeiro V, Fernandes T, Vassallo DV, Oliveira EM, Salaices M: Aerobic exercise reduces oxidative stress and improves vascular changes of small mesenteric and coronary arteries in hypertension. Br J Pharmacol 2013;168:686703.

44 Kimura H, Kon N, Furukawa S, Mukaida M, Yamakura F, Matsumoto K, Sone H, Murakami-Murofushi K: Effect of endurance exercise training on oxidative stress in spontaneously hypertensive rats (SHR) after emergence of hypertension. Clin Exp Hypertens 2010;32:407-415.

-45 Rush JW, Turk JR, Laughlin MH: Exercise training regulates SOD-1 and oxidative stress in porcine aortic endothelium. Am J Physiol Heart Circ Physiol 2003;284:H1378-R1387. 